\title{
Article
}

\section{In Vacuo Dispersion-Like Spectral Lags in Gamma-Ray Bursts}

\author{
Giovanni Amelino-Camelia ${ }^{1, *}$, Giacomo D'Amico ${ }^{2}$, Fabrizio Fiore ${ }^{3}$, Simonetta Puccetti ${ }^{4}$ and Michele Ronco ${ }^{5}$ \\ 1 Dipartimento di Fisica Ettore Pancini, Università di Napoli “Federico II", and INFN, Sezione di Napoli, \\ Complesso University Monte S. Angelo, I-80126 Napoli, Italy \\ 2 Department of Physics and Technology, University of Bergen, 5020 Bergen, Norway; giacomo.damico@uib.no \\ 3 INAF-Osservatorio Astronomico di Trieste, via G.B. Tiepolo 11, 34143 Trieste, Italy; fabrizio.fiore@inaf.it \\ 4 Agenzia Spaziale Italiana, Unità di Ricerca Scientifica, Via del Politecnico, 00133 Roma, Italy; \\ simonetta.puccetti@asdc.asi.it \\ 5 Dipartimento di Fisica, Università di Roma “La Sapienza”, P.le A. Moro 2, 00185 Roma, Italy; \\ michele.ronco@uniroma1.it \\ * Correspondence: giovanni.amelinocamelia@unina.it
}

check for updates

Citation: Amelino-Camelia, G.;

D’Amico, G.; Fiore, F.; Puccetti, S.;

Ronco, M. In Vacuo Dispersion-Like Spectral Lags in Gamma-Ray Bursts. Symmetry 2021, 13, 541. https:// doi.org/10.3390/sym13040541

Academic Editors: Ángel Ballesteros and Sergei D. Odintsov

Received: 21 January 2021

Accepted: 12 March 2021

Published: 25 March 2021

Publisher's Note: MDPI stays neutral with regard to jurisdictional claims in published maps and institutional affiliations.

Copyright: (c) 2021 by the authors. Licensee MDPI, Basel, Switzerland. This article is an open access article distributed under the terms and conditions of the Creative Commons Attribution (CC BY) license (https:// creativecommons.org/licenses/by/ $4.0 /)$.

\begin{abstract}
Some recent studies exposed preliminary but rather intriguing statistical evidence of in vacuo dispersion-like spectral lags for gamma-ray bursts (GRBs), a linear correlation between time of observation and energy of GRB particles, which is expected in some models of quantum geometry. Those results focused on testing in vacuo dispersion for the most energetic GRB particles, and in particular only included photons with energy at emission greater than $40 \mathrm{GeV}$. We here extend the window of the statistical analysis down to $5 \mathrm{GeV}$ and find results that are consistent with what had been previously noticed at higher energies.
\end{abstract}

Keywords: quantum groups; quantum gravity phenomenology; gamma-ray bursts

\section{Introduction}

Over the last 15 years, there has been considerable interest (see e.g., Refs. [1-10] and references therein) in descriptions of spacetime based on quantum geometry that produce in vacuo dispersion, the possibility that spacetime itself might behave essentially like a dispersive medium for particle propagation: there might be an energy dependence of the travel times of ultrarelativistic particles from a given source to a given detector. This is the most studied scenario within a broader research program [1] concerning the possibility that relativistic symmetries might be affected by quantum-gravity effects (A partly related research program is known as the SME (Standard Model Extension), a framework considering all possible (with or without "quantum-gravity motivation") Lorentz-violating modifications of the Standard Model of particle physics [11]. The SME phenomenology mainly focuses Ref. [12] on dimension-four operators added to the Standard-Model lagrangian, whereas the effects we here consider would be described in field-theory in terms of dimension-five operators. However, describing photons within the SME the effects of dispersion would be combined with birefringence [13-17], which we here assume to be absent consistently with results obtained in frameworks alternative to the SME, such as those studied in Refs. $[1,3,7]$ and references therein).

It is well established [1-4] that the analysis of GRBs could allow us to test the in vacuo dispersion hypothesis. Some of us were involved in the first studies using IceCube data for searching for GRB-neutrino in vacuo dispersion candidates [9,18-20]. Analogous investigations were performed in a series of studies [21-23] (also see [24]) focusing on the highest-energy GRB photons observed by the Fermi telescope. As summarized in Figure 1 these studies provided rather intriguing statistical evidence of in vacuo dispersion-like spectral lags. The values of $\Delta t$ used in Figure 1 reflect the difference between the time of observation of the relevant particle and the time of observation of the first low-energy peak 
in the GRB to which the particle might be associated. The values of $E^{*}$ used in Figure 1 are obtained from the formula

$$
E^{*} \equiv E \frac{D(z)}{D(1)}
$$

where $z$ is the redshift of the relevant GRB and

$$
D(z)=\int_{0}^{z} d \zeta \frac{(1+\zeta)}{H_{0} \sqrt{\Omega_{\Lambda}+(1+\zeta)^{3} \Omega_{m}}} .
$$

denoting, as usual, with $\Omega_{\Lambda}, H_{0}$ and $\Omega_{m}$ respectively the cosmological constant, the Hubble parameter and the matter fraction, for which we take the values given in Ref. [25].

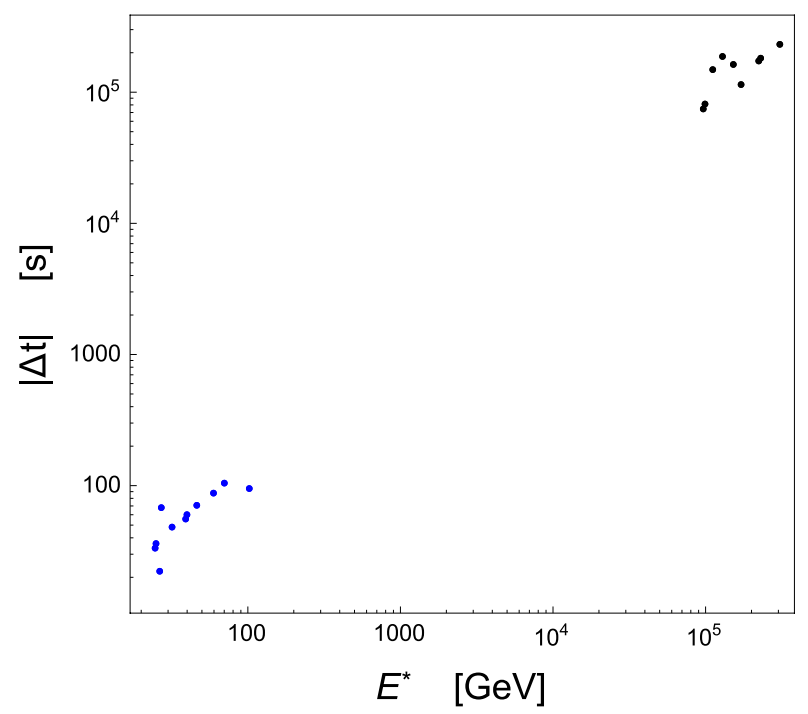

Figure 1. Values of $|\Delta t|$ versus $E^{*}$ for the IceCube GRB-neutrino candidates discussed in Refs. $[18,20]$ (black points) and for the GRB photons discussed in Refs. [20,23] (blue points). The photon points in figure also factor in the result of a one-parameter fit estimating the average magnitude of intrinsic time lags (details in Refs. [20,23]).

The most studied [1-10] model of quantum-gravity-induced in vacuo dispersion is (This formula for $\Delta t$ is expected to arise from Planck-scale modifications of the dispersion relation [4-6] and such modifications of the dispersion relation would affect many aspects of physics, with however effects that are irrelevantly small [1] in nearly all experimental context, these astrophysical time-of-travel studies being one rare exception in which, thanks to the huge amplification provided by the travel times, we might have a chance to test the effects.)

$$
\Delta t=\eta_{X} \frac{E}{M_{P}} D(z) \pm \delta_{X} \frac{E}{M_{P}} D(z),
$$

which in terms of $E^{*}$ takes the form

$$
\Delta t=\eta_{X} D(1) \frac{E^{*}}{M_{P}} \pm \delta_{X} D(1) \frac{E^{*}}{M_{P}} .
$$

$M_{P}$ denotes the Planck scale $\left(\simeq 1.2 \cdot 10^{28} \mathrm{eV}\right)$ and the values of the parameters $\eta_{\mathrm{X}}$ and $\delta_{X}$ in (3) are to be determined experimentally. In (3) the notation " $\pm \delta_{X}$ " reflects the fact that $\delta_{X}$ parametrizes the size of quantum-uncertainty (fuzziness) effects. Instead the parameter $\eta_{X}$ characterizes systematic effects: for example in our conventions for positive $\eta_{X}$ and $\delta_{X}=0$ a high-energy particle is detected systematically after a low-energy particle (if the two particles are emitted simultaneously). The label $X$ for $\delta_{X}$ and $\eta_{X}$ intends to allow for a possible dependence $[1,10]$ of these parameters on the type of particles (so that for 
example for neutrinos and photons one would have $\eta_{v}, \delta_{v}, \eta_{\gamma}, \delta_{\gamma}$ ) and in principle also on spin/helicity (so that for example for neutrinos one would have $\eta_{v+}, \delta_{v+}, \eta_{v-}, \delta_{v-}$ ).

The black points in Figure 1 are "GRB-neutrino candidates" in the sense of Ref. [18], while the blue points correspond to GRB photons with energy at emission greater than $40 \mathrm{GeV}$. The linear correlation between $\Delta t$ and $E^{*}$ visible in Figure 1 is just of the type expected for quantum-gravity-induced in vacuo dispersion. It might of course be accidental, but it has been estimated [18] that for the relevant GRB-neutrino candidates such a high level of correlation would occur accidentally only in less than 1\% of cases, while GRB photons could produce such high correlation (in absence of in vacuo dispersion) only in less than $0.1 \%$ of cases [20]. The "statistical evidence" summarized in Figure 1 is evidently intriguing enough to motivate us to explore whether or not the in vacuo dispersion-like spectral lags persist at lower energies.

\section{Analysis}

One challenge for this is that evidently we cannot simply apply to lower-energy photons the reasoning which led to Figure 1: as stressed above the $\Delta t$ in Figure 1 is the difference between the time of observation of the relevant particle and the time of observation of the first low-energy peak in the GRB, so it is a $\Delta t$ which makes sense for in vacuo dispersion studies only for photons which one might think were emitted in (near) coincidence with the first peak of the GRB. This assumption is (challengeable [26] but) plausible [23] for the few highest-energy GRB photons relevant for Figure 1, with energy at emission greater than $40 \mathrm{GeV}$, but of course it cannot apply to all photons in a GRB. Conceptually the main aspect of novelty of our analysis concerns a strategy for handling this challenge.

We consider the same GRBs relevant for the analysis summarized in Figure 1, but now including all photons from those GRBs with energy at the source greater than $5 \mathrm{GeV}$, thereby lowering the cutoff by nearly an order of magnitude. Only 11 photons took part in the previous analyses whose findings were summarized in our Figure 1, whereas the analysis we are here reporting involves a total of 148 photons. For the reasons discussed above, we do not consider the $\Delta t$ (with reference to the first peak of the GRB), but rather we consider a $\Delta t_{\text {pair }}$, which gives for each pair of photons in our sample their difference of time of observation. Essentially each pair of photons (from the same GRB) in our sample is taken to give us an estimated value of $\eta_{\gamma}$, by simply computing

$$
\eta_{\gamma}^{[p a i r]} \equiv \frac{M_{P} \Delta t_{\text {pair }}}{D(1) E_{\text {pair }}^{*}}
$$

where $E_{\text {pair }}^{*}$ is the difference in values of $E^{*}$ for the two photons in the pair. Of course the $\Delta t_{\text {pair }}$ for many pairs of photons in our sample could not possibly have anything to do with in vacuo dispersion: if the two photons were produced from different phases of the GRB (different peaks) their $\Delta t_{\text {pair }}$ will be dominated by the intrinsic time-of-emission difference. Those values of $\eta_{\gamma}^{[\text {pair }]}$ will be spurious, they will be "noise" for our analysis. However we also of course expect that some pairs of photons in our sample were emitted nearly simultaneously, and for those pairs the $\Delta t_{\text {pair }}$ could truly estimate $\eta_{\gamma}$. Since estimating $\eta_{\gamma}$ from the photons in Figure 1 one gets $\eta_{\gamma}=30 \pm 6$, the preliminary evidence here summarized in Fig.1 would find additional support if this sort of analysis showed that values of $\eta_{\gamma}^{[\text {pair }]}$ of about 30 are surprisingly frequent, more frequent than expected without a relationship between arrival times and energy of the type produced by in vacuo dispersion.

This is just what we find, as shown perhaps most vividly by the content of Figure 2, which was obtained including in the analysis all possible pairs of photons belonging to the same GRB. The main point to be noticed in Figure 2 is that we find in our sample a frequency of occurrence of values of $\eta_{\gamma}^{[\text {pair }]}$ between 25 and 35 which is tangibly higher than one would have expected in absence of a correlation between $\Delta t_{\text {pair }}$ and $E_{\text {pair }}^{*}$. Following a standard 
strategy of analysis (see, e.g., Refs. [24,27]) we estimate how frequently $25 \leq \eta_{\gamma}^{[\text {pair }]} \leq 35$ should occur in absence of correlation between $\Delta t_{\text {pair }}$ and $E_{\text {pair }}^{*}$ by producing $10^{5}$ sets of simulated data, each obtained by reshuffling randomly the times of observation of the photons in our sample. More details on this and other aspects of our analysis are given in Appendix A. Also in Appendix A we show that our findings are strikingly robust with respect to restricting the analysis to only part of our data set: values of $\eta_{\gamma}^{[\text {pair }]}$ between 25 and 35 occur at a rate higher than expected for all meaningful portions of our data set. Most notably, values of $\eta_{\gamma}^{[\text {pair }]}$ between 25 and 35 occur at a rate higher than expected even if we exclude from the analysis the photons whose energy at emission is greater than $40 \mathrm{GeV}$ (the photons that were taken into account in the analyses leading to the content of our Figure 1).

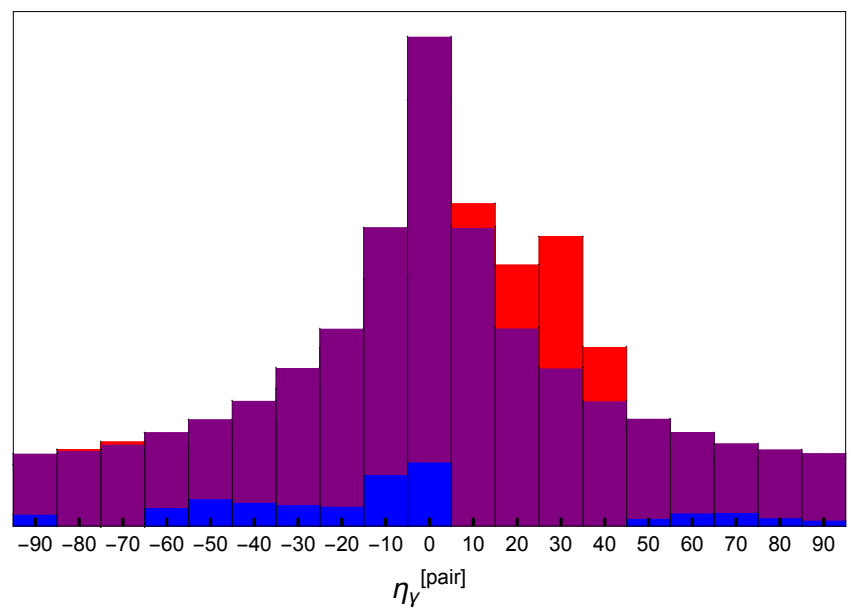

Figure 2. Normalized distribution of $\eta_{\gamma}^{[\text {pair }]}$ for all pairs of photons (from the same GRB) within our data set. For bins where the observed population is higher than expected we color the bar in purple up to the level expected, showing then the excess in red. For bins where the observed population is lower than expected the bar height gives the expected population, while the blue portion of the bar quantifies the amount by which the observed population is lower than expected.

It is also noteworthy that we find (see Appendix A) that an excess of results for $\eta_{\gamma}^{[\text {pair }]}$ between 25 and 35 as big as shown by our data should occur accidentally (in absence of in vacuo dispersion) in less than $0.5 \%$ of cases.

Also intriguing is the content of our Figure 3, which offers an intuitive characterization of the consistency that emerged from our analysis between what had been found in previous studies of GRB photons with energy at emission greater than $40 \mathrm{GeV}$, and what we now find for GRB photons with energy between 5 and $40 \mathrm{GeV}$.

\section{Closing Remarks}

We used data that were already available at the time of the studies that led to Figure 1 (which in particular focused on photons with energy at emission greater than $40 \mathrm{GeV}$ ) but nobody had looked before at those data for photons with energy at emission between 5 and $40 \mathrm{GeV}$, from the perspective of Figure 1. Our findings should therefore be viewed as providing further motivation for investigating this scenario. Of course, if this feature is confirmed by future studies the next task would be to establish whether it is truly connected with quantum-gravity-induced in vacuo dispersion, rather than being related to some intrinsic property of GRB signals. Within our analysis the imprint of in vacuo dispersion is coded in the $D(z)$ for the distance dependence and, while that does give a good match to the data, one should keep in mind that only a few redshifts (a few GRBs) were relevant for our analysis. If we are actually seeing some form of in vacuo dispersion it would most likely be of statistical ("fuzzy") nature since other studies have provided evidence strongly disfavoring the possibility that this type of in vacuo dispersion effects would affect systematically all photons [28]. 


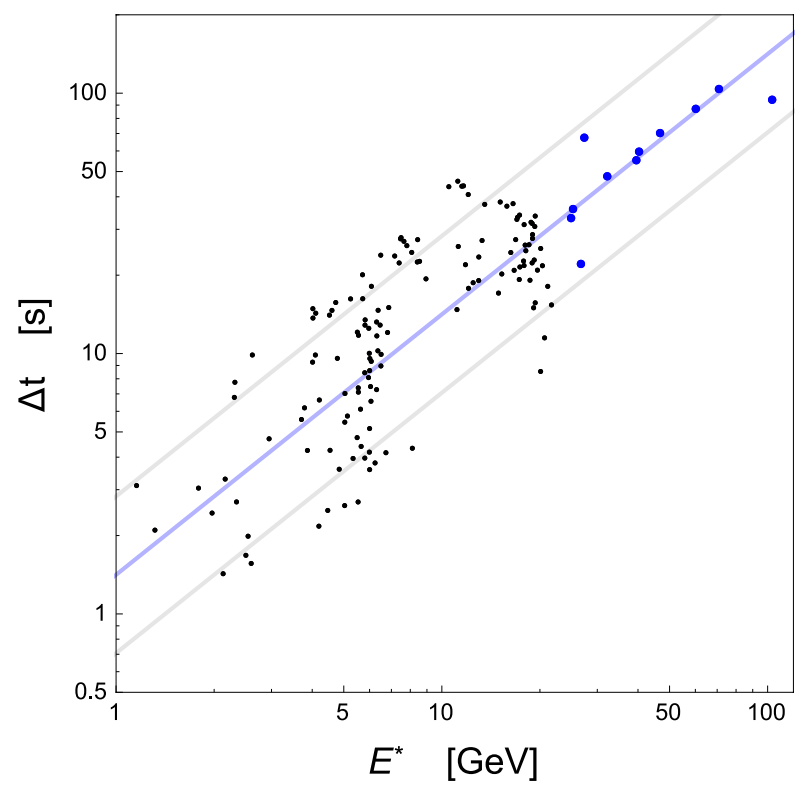

Figure 3. As in Figure 1 blue points here are for the GRB photons discussed in Refs. [20,23] (with energy at emission greater than $40 \mathrm{GeV}$ ). Here black points give the $E_{\text {pair }}^{*}$ and the $\Delta t_{\text {pair }}$ for our pairs of GRB photons, including only cases in which both photons have energy at emission lower than $40 \mathrm{GeV}$ and the associated value of $\eta_{\gamma}^{[\text {pair }]}$ is rather sharp (relative error of less than $30 \%$ ) and between 10 and 100. The gray lines characterize the range of values of $\eta_{\gamma}$ favored by the blue points, which is also the region where black points are denser. The violet line is for $\eta_{\gamma}=34$ and intends to help the reader notice the similarity of statistical properties between the distribution of black and blue points, that goes perhaps even beyond the quantitative aspects exposed in our histograms.

Author Contributions: All authors contributed equally to research and writing the manuscript.

Funding: G.A.-C.'s work on this project was supported by the FQXi grant 2018-190483 and by the MIUR PRIN 2017 grant 20179ZF5KS.

Institutional Review Board Statement: Not applicable.

Informed Consent Statement: Not applicable.

Data Availability Statement: Not applicable.

Conflicts of Interest: The authors declare no conflict of interest.

\section{Appendix A}

In this appendix we provide further details on the results discussed in the main text and also discuss some additional corollary results.

Our analysis focuses on the same GRBs whose photons took part in the analyses which led to the picture here summarized in Figure 1. These are the GRBs that provide us the full range of energies relevant for our analysis, including some photons with energy at emission greater than $40 \mathrm{GeV}$ : GRB080916C, GRB090510, GRB090902B, GRB090926A, GRB100414A, GRB130427A, GRB160509A. The relevant data were downloaded from the Fermi-LAT archive and they were calibrated and cleaned using the LAT ScienceTools-v10r0p5 package, which is available from the Fermi Science Support Center.

For reasons that shall soon be clear it was valuable for us to divide our data sample in different subgroups, characterized by different ranges of values for the energy at emission, which we denote by $E_{0}$. We label as "high" the photons in our sample with $E_{0}>40 \mathrm{GeV}$, with "medium" those with $15 \mathrm{GeV} \leq E_{0} \leq 40 \mathrm{GeV}$, and with "low" those with $5 \mathrm{GeV}$ $\leq E_{0} \leq 15 \mathrm{GeV}$. Our "high" photons were already taken into account in the previous studies which led to Figure 1, so it is particularly valuable to keep them distinct from the other photons in our sample (the ones we label as "medium" and "low"). 
Let us start with the content of Figure 2, which takes into account all pairs of photons (of course from the same GRB) within our data set. Each such pair typically contributes to more than one of our bins, considering that the energies of the photons are not known very precisely. The contribution of a given pair to each bin is computed generating a gaussian distribution with mean value $\eta_{\gamma}$ (calculated with Equation (5)) and standard deviation $\sigma_{\gamma}$ obtained by error propagation of the energy uncertainty, which we assume to be of $10 \%$. Then, we compute the area of this distribution, which we limit in the interval $\left[\eta_{\gamma}-\sigma_{\eta}, \eta_{\gamma}+\sigma_{\eta}\right]$, falling within each bin, in order to evaluate the value to assign to a given bin. Thus, each pair in general contributes to more than one bin and does that with a gaussian weight. The expected frequency of occurrence of values of $\eta_{\gamma}^{[\text {pair }]}$ corresponding to a given bin was estimated by producing $10^{5}$ sets of simulated data, each obtained by reshuffling randomly the times of observation of the photons (of each GRB) in our sample. Of particular significance for our objective is the higher than expected observed frequency of values of $\eta_{\gamma}^{[\text {pair }]}$ between 25 and 35. Interestingly, we find, using our simulated data obtained by time reshuffling, that the excess in bin $25 \leq \eta_{\gamma}^{[\text {pair }]} \leq 35$ visible in Figure 2 is expected to occur accidentally only in $1.2 \%$ of cases.

In Figure A1 we report the results of an analysis that is just like the analysis that produced Figure 2 but now excludes the contributions from the "high" photons (with energy at emission greater than $40 \mathrm{GeV}$ ). It is noteworthy that one still has a higher than expected observed frequency of values of $\eta_{\gamma}^{[\text {pair }]}$ between 25 and 35, and for this case we estimate, using our simulated data obtained by time reshuffling, that the excess of occupancy of the bin $25 \leq \eta_{\gamma}^{[\text {pair }]} \leq 35$ visible in Figure A1 should occur accidentally only in $0.6 \%$ of cases.

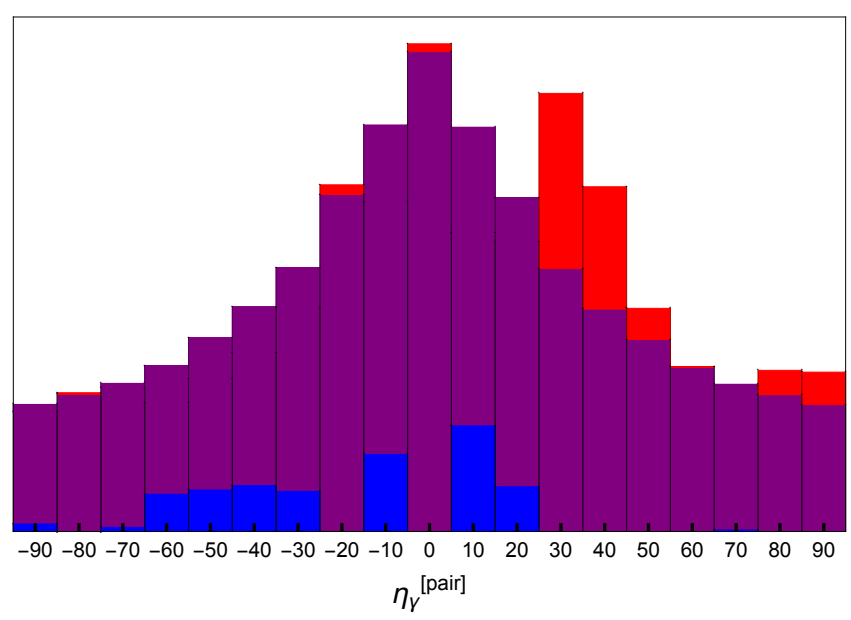

Figure A1. Results of a study of the type already described in the previous Figure 2, but now taking into account only pairs of photons that do not involve a "high" photon. Color coding of the bars is the same as for Figure 2.

It is noteworthy that a higher than expected observed frequency of values of $\eta_{\gamma}^{[\text {pair }]}$ between 25 and 35 is present also if we constrain the two photons in a pair to be of different type, for what concerns our categories of "high", "medium" and "low". In Figure A2 we show the results we obtain for pairs composed of a "medium" (15 GeV $\left.\leq E_{0} \leq 40 \mathrm{GeV}\right)$ and a "low" ( $\left.5 \mathrm{GeV} \leq E_{0} \leq 15 \mathrm{GeV}\right)$ photon. For this case we estimate, using our simulated data obtained by time reshuffling, that the excess of occupancy of the bin $25 \leq \eta_{\gamma}^{[\text {pair }]} \leq 35$ visible in Figure A2 should occur accidentally only in $0.2 \%$ of cases. 


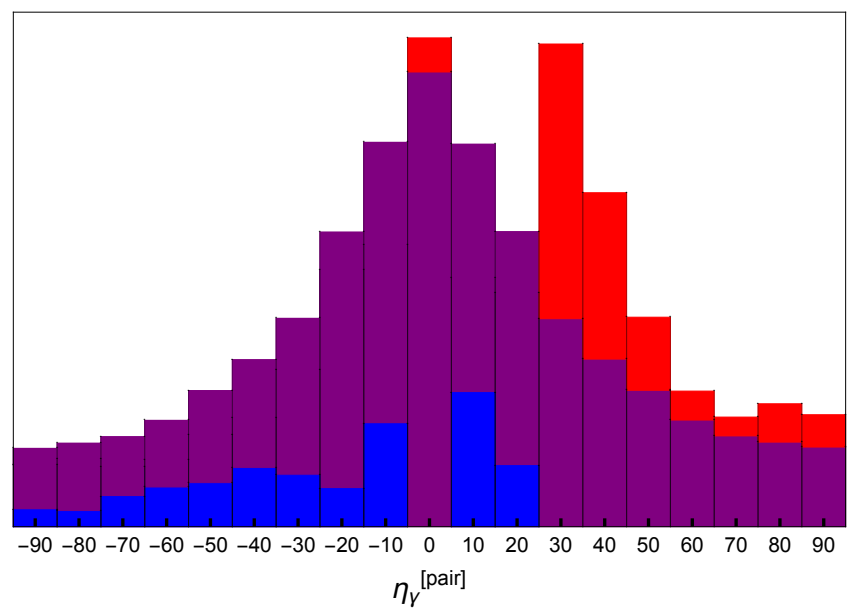

Figure A2. Here we show the same type of results already shown in Figures 2 and A2, but now taking into account only pairs composed of a "medium" and a "low" photon.

In Figure 3 we show the results we obtain for pairs composed of a "high" $\left(E_{0}>40 \mathrm{GeV}\right)$ and a "low" ( $\left.5 \mathrm{GeV} \leq E_{0} \leq 15 \mathrm{GeV}\right)$ photon. As visible in Figure A3, once again we find a higher than expected observed frequency of values of $\eta_{\gamma}^{[\text {pair }]}$ between 25 and 35 , even though in this case the statistical significance is less striking: using our simulated data obtained by time reshuffling, we find that the excess of occupancy of the bin $25 \leq \eta_{\gamma}^{[\text {pair }]} \leq 35$ visible in Figure A3 should occur accidentally in about $14 \%$ of cases (though this result reflects in part also the fact that we do not have high statistics of high-low pairs).

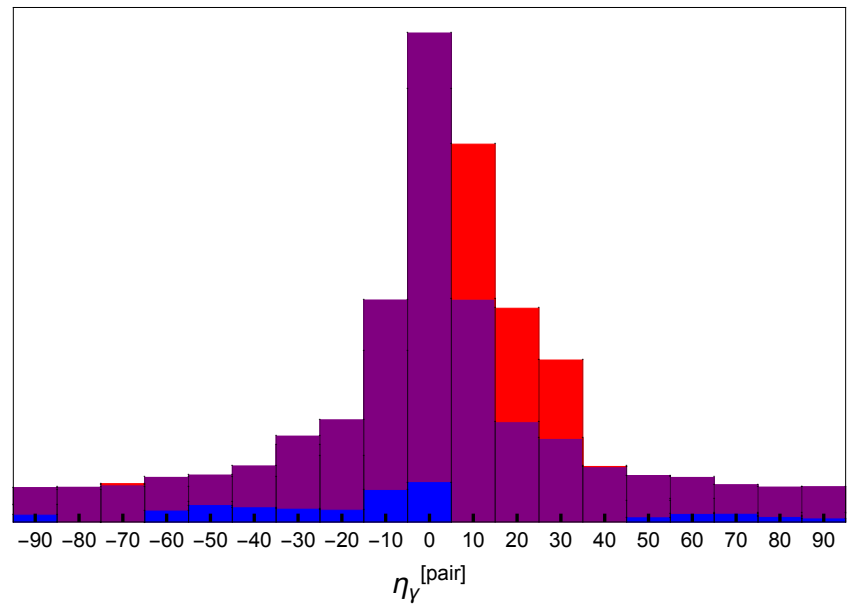

Figure A3. Results of a study of the type already described in the previous Figures 2, A1 and A2, but now we require the pair to be made of a "high" and a "low" photon.

Between the main text and this appendix we discussed a total of four analyses which are to a large extent independent (though not totally independent). Each analysis uses different pairs, but for example the results reported in Figures A2 and A3 could be used to anticipate to some extent the results of Figure 2. Considering the (rather high) level of independence of the different analyses it is striking that in all cases we found an excess of results with $\eta_{\gamma}$ between 25 and 35 . We found that three of our analyses have significance between $0.2 \%$ and $1.2 \%$, while the fourth analysis has significance of about $14 \%$. The present data situation is surely intriguing, but dwelling on percentages is in our opinion premature. We therefore prudently quote in the main text an overall significance of about $0.5 \%$. 


\section{References}

1. Amelino-Camelia, G. Quantum Spacetime Phenomenology. Living Rev. Relativ. 2013, 16, 5. [CrossRef] [PubMed]

2. Jacob, U.; Piran, T. Neutrinos from gamma-ray bursts as a tool to explore quantum-gravity-induced Lorentz violation. Nat. Phys. 2007, 3, 87. [CrossRef]

3. Amelino-Camelia, G.; Smolin, L. Prospects for constraining quantum gravity dispersion with near term observations. Phys. Rev. D 2009, 80, 084017. [CrossRef]

4. Amelino-Camelia, G.; Ellis, J.; Mavromatos, N.E.; Nanopoulos, D.V.; Sarkar, S. Tests of quantum gravity from observations. Nature 1998, 393, 763. [CrossRef]

5. Gambini, R.; Pullin, J. Nonstandard Optics from Quantum Space-Time. Phys. Rev. 1999, D59, 124021. [CrossRef]

6. Alfaro, J.; Morales-Tecotl, H.A.; Urrutia, L.F. Quantum Gravity Corrections to Neutrino Propagation. Phys. Rev. Lett. 2000, 84, 2318. [CrossRef]

7. Amelino-Camelia, G.; Majid, S. Waves on noncommutative space-time and gamma-ray bursts. Int. J. Mod. Phys. A 2000, 15, 4301. [CrossRef]

8. Myers, R.C.; Pospelov, M. Ultraviolet modifications of dispersion relations in effective field theory. Phys. Rev. Lett. 2003, 90, 211601. [CrossRef]

9. Amelino-Camelia, G.; Guetta, D.; Piran, T. Icecube Neutrinos and Lorentz Invariance Violation. Astrophys. J. 2015, 806, 269. [CrossRef]

10. Stecker, F.W.; Scully, S.T.; Liberati, S.; Mattingly, D. Searching for traces of Planck-scale physics with high energy neutrinos. Phys. Rev. D 2015, 91, 045009. [CrossRef]

11. Colladay, D.; Kostelecky, V.A. Lorentz-violating extension of the standard model. Phys. Rev. D 1998, 58, 116002. [CrossRef]

12. Kostelecky, V.A.; Russell, N. Data Tables for Lorentz and CPT Violation. arXiv 2008, arXiv:0801.0287.

13. Friedman, A.S.; Gerasimov, R.; Leon, D.; Stevens, W.; Tytler, D.; Keating, B.G.; Kislat, F. Improved constraints on anisotropic birefringent Lorentz invariance and CPT violation from broadband optical polarimetry of high redshift galaxies. Phys. Rev. D 2020, 102, 043008. [CrossRef]

14. Pogosian, L.; Shimon, M.; Mewes, M.; Keating, B. Future CMB constraints on cosmic birefringence and implications for fundamental physics. Phys. Rev. D 2019, 100, 023507. [CrossRef]

15. Friedman, S.; Leon, D.; Crowley, K.D.; Johnson, D.; Teply, G.; Tytler, D.; Keating, B.G.; Cole, G.M. Constraints on Lorentz invariance and CPT violation using optical photometry and polarimetry of active galaxies BL Lacertae and S5. Phys. Rev. D 2019, 99, 035045. [CrossRef]

16. Kislat, F. Constraints on Lorentz Invariance Violation from Optical Polarimetry of Astrophysical Objects. Symmetry 2018, 10, 596. [CrossRef]

17. Kislat, F.; Krawczynski, H. Planck-scale constraints on anisotropic Lorentz and CPT invariance violations from optical polarization measurements. Phys. Rev. D 2017, 95, 083013. [CrossRef]

18. Amelino-Camelia, G.; Barcaroli, L.; D’Amico, G.; Loret, N.; Rosati, G. IceCube and GRB neutrinos propagating in quantum spacetime. Phys. Lett. B 2016, 761, 318. [CrossRef]

19. Amelino-Camelia, G.; Barcaroli, L.; D'Amico, G.; Loret, N.; Rosati, G. Quantum-gravity-induced dual lensing and IceCube neutrinos. arXiv 2016, arXiv:1609.03982 [gr-qc].

20. Amelino-Camelia, G.; D’Amico, G.; Rosati, G.; Loret, N. In-vacuo-dispersion features for GRB neutrinos and photons. Nat. Astron. 2017, 1, 0139. [CrossRef]

21. Zhang, S.; Ma, B.Q. Lorentz violation from gamma-ray bursts. Astropart. Phys. 2014, 61, 108. [CrossRef]

22. Xu, H.; Ma, B.Q. Light speed variation from gamma-ray bursts. Astropart. Phys. 2016, 82, 72. [CrossRef]

23. Xu, H.; Ma, B.Q. Light speed variation from gamma ray burst GRB 160509A. Phys. Lett. B 2016, 760, 602. [CrossRef]

24. Amelino-Camelia, G.; Fiore, F.; Guetta, D.; Puccetti, S. Quantum-spacetime scenarios and soft spectral lags of the remarkable GRB130427A. Adv. High Energy Phys. 2014, 2014, 597384. [CrossRef]

25. Planck Collaboration: Ade, P.A.R.; Aghanim, N.; Arnaud, M.; Ashdown, M.; Aumont, J.; Baccigalupi, C.; Banday, A.J.; Bartlett, J.G.; Bartolo, N.; Battaner, E.; et al. Planck 2015 results. XIII. Cosmological parameters. arXiv 2016, arXiv:1502.01589v2.

26. Ghirlanda, G.; Ghisellini, G.; Nava, L. The onset of the GeV afterglow of GRB 090510. Astron. Astrophys. 2010, 510, L7. [CrossRef]

27. Vasileiou, V.; Jacholkowska, A.; Piron, F.; Bolmont, J.; Couturier, C.; Granot, J.; Stecker, F.W.; Cohen-Tanugi, J.; Longo, F. Constraints on Lorentz invariance violation from Fermi-Large Area Telescope observations of gamma-ray bursts. Phys. Rev. D 2013, 87, 122001. [CrossRef]

28. Abdo, A.A.; Ackermann, M.; Ajello, M.; Asano, K.; Atwood, W.B.; Axelsson, M.; Baldini, L.; Ballet, J.; Barbiellini, G.; Baring, M.G. A limit on the variation of the speed of light arising from quantum gravity effects. [Fermi LAT/GBM Collaborations]. Nature 2009, 462, 331. [CrossRef] 